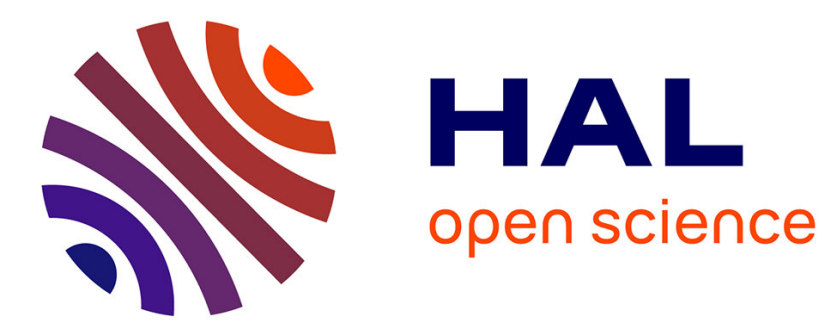

\title{
Using Activation Energies to Elucidate Mechanisms of Water Dynamics
}

\author{
Zeke A Piskulich, Damien Laage, Ward H Thompson
}

\section{To cite this version:}

Zeke A Piskulich, Damien Laage, Ward H Thompson. Using Activation Energies to Elucidate Mechanisms of Water Dynamics. Journal of Physical Chemistry A, 2021. hal-03420260

\section{HAL Id: hal-03420260 \\ https: / hal.sorbonne-universite.fr/hal-03420260}

Submitted on 9 Nov 2021

HAL is a multi-disciplinary open access archive for the deposit and dissemination of scientific research documents, whether they are published or not. The documents may come from teaching and research institutions in France or abroad, or from public or private research centers.
L'archive ouverte pluridisciplinaire HAL, est destinée au dépôt et à la diffusion de documents scientifiques de niveau recherche, publiés ou non, émanant des établissements d'enseignement et de recherche français ou étrangers, des laboratoires publics ou privés. 


\title{
Using Activation Energies to Elucidate Mechanisms of Water Dynamics
}

\author{
Zeke A. Piskulich, ${ }^{\dagger}$, Damien Laage, ${ }^{*, \dagger}$ and Ward H. Thompson ${ }^{*, \dagger}$ \\ $\dagger$ Department of Chemistry, University of Kansas, Lawrence, KS 66045, USA \\ $\ddagger$ PASTEUR, Department de Chimie, École Normale Supérieure, PSL University, Sorbonne \\ Université, CNRS, Paris, 75005, France \\ ФCurrent address: Department of Chemistry, Boston University, Boston, MA, 02215
}

E-mail: damien.laage@ens.psl.eu; wthompson@ku.edu

October 15, 2021

\section{Abstract}

Recent advances in the calculation of activation energies are shedding new light on the dynamical timescales of liquid water. In this Perspective, we examine how activation energies elucidate the central, but not singular, role of the exchange of hydrogen-bond (H-bond) partners that rearranges the $\mathrm{H}$-bond network of water. The contributions of other motions to dynamical timescales and their associated activation energies are discussed along with one case, vibrational spectral diffusion, where $\mathrm{H}$-bond exchanges are not mechanistically significant. Nascent progress on outstanding challenges, including descriptions of non-Arrhenius effects and activation volumes, are detailed along with some directions for future investigations.

\section{Introduction}

The dynamics of liquids can be characterized in myriad ways, including e.g., self-diffusion coefficient, reorientation time, dielectric relaxation time, and viscosity. While all of these properties probe different behaviors of the liquid, some of them are expected to share a common molecular origin and result from the same elementary structural rearrangements. A central paradigm in chemistry is indeed that function follows structure, and the corollary is that dynamics is determined by the energetics associated with changes between structures. In the context of water, this dictates that the behavior of the liquid is determined by the disordered hydrogen-bonding (H-bonding) network that represents its key structural feature. That this is so is evident in many of the unique (or at least rare) properties of water, e.g., a density maximum with temperature, isotopic effects in the liquid thermodynamics, a liquidliquid phase transition for the supercooled liquid, solvation properties, etc. The rearrangements of this H-bond network, and in particular the exchange of $\mathrm{H}$-bond partners that changes the H-bond network connectivity, are therefore expected to be the central elementary step underlying (most) dynamical timescales in liquid water. This connection is intuitive and forms the implicit basis for much of the discussions in the literature of liquid water dynamics. ${ }^{1-3}$

However, the relationship between structure and dynamics is also fraught with imprecision. The relaxation time of a quantity is determined not only by the characteristic time of the structural rearrangements but also by the amplitude of the change in that quantity that is induced by each elementary rearrangement. In addition, multiple structural rearrangements can affect the same quantity. This can be illustrated for one water dynamical timescale, $\mathrm{OH}$ reori- 
entation time, for which the connection with $\mathrm{H}$-bond exchange times has been made theoretically precise. ${ }^{4,5}$ This description showed that other factors - the angle the $\mathrm{OH}$ bond traverses during an exchange, reorientation of the intact H-bonded pair between exchanges - also contribute to the $\mathrm{OH}$ reorientation time, thus illustrating the challenges in applying intuitive, but often overly simplified conceptual interpretations, as discussed in detail in Sec. 3. More broadly, key questions remain about how Hbond exchange times are manifested in various other dynamical timescales such as the self-diffusion coefficient, viscosity, spectral diffusion, dielectric relaxation, and solvation dynamics, which currently lack the rigorous theoretical connection available for reorientation.

Connecting a dynamical timescale to a structural rearrangement and its associated energetic barrier is not straightforward. This can be shown by considering the simple expression of a rate constant, $k(T)$, in terms of the transition state theory (TST) result, ${ }^{6,7} k_{T S T}(T)$, and the associated transmission coefficient, $\kappa(T)$,

$$
\begin{aligned}
k(T) & =\kappa(T) k_{T S T}(T), \\
& =\kappa(T) \frac{k_{B} T}{h} \frac{Q^{\ddagger}(T)}{Q_{r}(T)} e^{-\beta E^{\ddagger}}, \\
& =\kappa(T) \nu_{s} e^{-\beta \Delta A^{\ddagger}(T)} .
\end{aligned}
$$

Here, $\beta=1 / k_{B} T$ with $k_{B}$ Boltzmann's constant, $h$ is Planck's constant, $Q_{r}$ and $Q^{\ddagger}$ are the reactant and transition state (TS) partition functions, $E^{\ddagger}$ and $\Delta A^{\ddagger}$ are the electronic energy and (Helmholtz) free energy differences between the TS and reactant configurations, and $\nu_{s}=\omega_{s} / 2 \pi$ is the reactant frequency in the reaction coordinate, $s$. This equation relates the dynamical timescale, $k^{-1}$, to the free energy barrier, $\Delta A^{\ddagger}$, associated with the underlying structural rearrangement. However, while $k(T)$ is measurable and thus independent of any choice of ours, each factor on the right-handside of eq. 1 depends on our choice of the reaction coordinate through the definition of the transition state. A first difficulty therefore lies in the identification of an adequate microscopic coordinate to describe the relevant structural rearrangement.

A second issue, even more critical in the present context, is that the timescale is not determined solely by the energetics determined by structure $\left(\Delta A^{\ddagger}\right)$ but includes contributions from the dynamical factors $\kappa$ and $\nu_{s} \cdot{ }^{5,8}$ One can assume there is an "exact" reaction coordinate for which $\kappa=1$ to leave only $\nu_{s}$ as a confounding factor. Assuming that $\nu_{s}$ is independent of $T,{ }^{9}$ eq. 1 indicates that $\Delta U^{\ddagger}$ can be obtained from the temperature dependence of $k$,

$$
\ln k(T)=\ln \nu_{s}-\frac{\Delta U^{\ddagger}}{k_{B} T}+\frac{\Delta S^{\ddagger}}{k_{B}},
$$

but $\Delta S^{\ddagger}$ can only be determined within a factor of $\ln \nu_{s}$.

Given these difficulties, how can the connection between the various dynamical timescales of water and H-bond exchanges be clarified? In this Perspective, we show that one powerful approach is through a careful comparison of their temperature dependences, typically characterized by the activation energy. Its application to liquid water provides a molecular understanding to the experimental observation ${ }^{3}$ that a broad range of dynamical quantities including, e.g., translational diffusion and reorientation - share similar activation energies, but also reveals why their activation energies are not strictly equal. In the following, we first introduce activation energies, their key role, and molecular interpretation. We show that their analysis for translational and rotational dynamics in liquid water reveals the central role played by H-bond exchanges in both processes. We then consider vibrational spectral diffusion in water and show that, in contrast to its traditional interpretation, activation energies establish that it is not caused by H-bond exchanges but rather by unsuccessful attempts at exchanges. Further, we show how this approach can be extended to open challenges involving non-Arrhenius processes and activation volumes, and we finally offer some concluding remarks. 


\section{The Central Role of Ac- tivation Energies}

The activation energy is defined as

$$
E_{a}=-\frac{\partial \ln k(T)}{\partial \beta},
$$

where, e.g., $k$ equals the inverse of a characteristic timescale or a rate constant for a chemical reaction. $E_{a}$ is unambiguously defined and accessible experimentally, in contrast with the potential energy barrier $\Delta U^{\ddagger}$ which depends on an arbitrary choice of reaction coordinate, as discussed above. It may not be obvious a priori that it is necessary to concern ourselves with $E_{a}$, or that the activation energy more clearly reveals the link between the various timescales (though certainly a comparison of both timescales and activation energies is naturally more revealing than one involving only the former). However, some further considerations strongly indicate that the activation energy might be a more fundamental property.

First, Tolman has shown that the activation energy for a chemical reaction can be rigorously expressed as

$$
E_{a}=\langle H\rangle_{\text {reacting }}-\langle H\rangle_{\text {reactant }},
$$

where $H$ is the system Hamiltonian and $\langle\cdots\rangle$ indicates a thermal average. Thus, $E_{a}$ is a measure of the average energy of species that react minus that of the reactant species. Note that in the case of no transition state recrossing, eq. 2, TST gives $E_{a}=\Delta U^{\ddagger}$, if one assumes that $\nu_{s}$ is temperature independent (the constant pressure activation energy would similarly yield $\Delta H^{\ddagger}$ under the same assumptions). This is consistent with the Tolman interpretation of the activation energy, eq. 4, which recognizes it as equal to the difference in internal energy of the reacting species and reactant species. In this context, if two timescales share similar activation energies, it implies that they not only exhibit similar responses to a change in temperature, but further suggests a common mechanistic component.

Second, as will be discussed in further detail in this section, for many dynamical quantities in liquid water, their temperature dependence is dominated by that of the structural rearrangement, e.g., the H-bond exchange for the reorientation time and diffusion coefficient, which is thermally activated. In contrast, the amplitudes of, e.g., the reorientational or translational displacement induced by each $\mathrm{H}$-bond exchange, change more weakly with temperature, and bring a smaller contribution to the overall activation energy. In other words, the activation energy as given by eq. 4 is expected to be less sensitive to the additional factors (e.g., the size of angular and translational jumps in reorientation and diffusion, respectively) that enter into the various timescales of water.

\subsection{An Example: Water Diffu- sion \& Reorientation}

These arguments are admittedly not conclusive, but can be more concretely explored by consideration and comparison of water reorientational and diffusive dynamics. Reorientation in water is most commonly characterized by the reorientational timescale $\tau_{2}$ that describes the longtime decay of the time correlation function,

$$
C_{2}(t)=\left\langle P_{2}\left[\vec{e}_{O H}(0) \cdot \vec{e}_{O H}(t)\right]\right\rangle,
$$

which is associated with $\mathrm{H}$-bond making and breaking; 4,5 here, $\vec{e}_{O H}(t)$ is the unit vector along the $\mathrm{OH}$ bond of a water molecule at time $t$ and $P_{2}$ is the second-order Legendre polynomial. Pump-probe anisotropy measurements ${ }^{10}$ can directly access $\tau_{2}$ and an average timescale $\left\langle\tau_{2}\right\rangle=\int_{0}^{\infty} C_{2}(t) d t$ can be determined from nuclear magnetic resonance (NMR) experiments. ${ }^{11}$ Diffusion is naturally characterized by the diffusion coefficient $D$ that is obtained from the long-time slope of the mean-squareddisplacement $(M S D)$,

$$
D=\lim _{t \rightarrow \infty} \frac{\left\langle|\vec{r}(t)-\vec{r}(0)|^{2}\right\rangle}{6 t} \equiv \lim _{t \rightarrow \infty} \frac{M S D(t)}{6 t},
$$

where $\vec{r}(t)$ is the position of a molecular site at time $t$. Note that $D$ is also obtainable from NMR measurements ${ }^{12}$ as well as quasi-elastic neutron scattering ${ }^{3,13}$ and tracer diffusion ex- 
periments. ${ }^{14}$

The activation energies associated with $\tau_{2}$ and $D$ can be obtained from a fluctuation theory for dynamics method ${ }^{15,16}$ that provides the analytical derivative of a rate constant (or timescale) with respect to temperature (as opposed to the numerical derivative obtained in an Arrhenius analysis).

The method has been described in detail elsewhere, ${ }^{15,16}$ but it can be readily understood using the example of the diffusion coefficient, $D$, given above in eq. 6. The temperature dependence in the $M S D$ comes from the Boltzmann sampling of coordinates and momenta of the $N$ atoms,

$$
M S D(t)=\frac{h^{-3 N}}{Q} \iint d \mathbf{p} d \mathbf{q} e^{-\beta H}|\vec{r}(t)-\vec{r}(0)|^{2},
$$

specifically the Boltzmann factor $\left(e^{-\beta H}\right)$ and the canonical partition function $(Q)$. It is straightforward to show that the derivative of the mean-squared-displacement with respect to $\beta$ is

$$
\begin{aligned}
\frac{\partial M S D(t)}{\partial \beta} & =-\left\langle\delta H(0)|\vec{r}(t)-\vec{r}(0)|^{2}\right\rangle \\
& \equiv-M S D_{H}(t),
\end{aligned}
$$

where $\delta H(0)=H(0)-\langle H\rangle$ is the fluctuation in the total system energy at time $t=0$ about its average value $\langle H\rangle$. The diffusion coefficient activation energy, $E_{a, D}$, is then given by

$$
E_{a, D}=\frac{\lim _{t \rightarrow \infty}\left\langle\delta H(0)|\vec{r}(t)-\vec{r}(0)|^{2}\right\rangle}{\lim _{t \rightarrow \infty}\left\langle|\vec{r}(t)-\vec{r}(0)|^{2}\right\rangle} .
$$

Note that, while this illustration is based on the diffusion coefficient, the method is applicable to any time-dependent - or, indeed, any time-independent - quantity. A key advantage of this fluctuation theory approach is that the activation energy can be calculated using this expression from simulations at a single temperature.

We have recently used this approach to examine many dynamical and equilibrium properties of water ${ }^{16-26}$ (and other systems ${ }^{27-29}$ ) including $\mathrm{OH}$ reorientation. ${ }^{17-21}$ and self- diffusion. ${ }^{17,20,21,24}$ One can use these results to carry out a simple thought experiment. If $\mathrm{OH}$ reorientation and diffusion (and other dynamics) in water is controlled by H-bond exchanges, the jump time should be the single characteristic of all of these dynamics. Moreover, such a relationship will extend to the corresponding activation energies (and other activation parameters, such as activation volume) that measure the energetics for each timescale that, within the assumptions of this thought experiment, are determined by the energetics of an H-bond exchange.

We can carry out this thought experiment by examining timescales and activation energies from a range of different water models. If the assumed centrality of the H-bond jumps is accurate, a single, clear relationship should exist between a given dynamical timescale and the jump time and, by extension, between the various timescales themselves.

The relevant comparisons suggested by this line of thinking are presented in Figs. 1 and 2. In particular, Fig. 1 shows the water selfdiffusion coefficient and the inverse $\mathrm{OH}$ reorientation time as a function of the rate constant for H-bond exchanges, $k_{0}=1 / \tau_{0}$, for nine different classical water models. (Note that the models include 3 - and 4-site rigid models, ${ }^{30-35}$ two 3 -site flexible models, ${ }^{34-36}$ and two models that include 3 -body interactions. ${ }^{37,38}$ For reference, the measured diffusion coefficient ${ }^{12}$ is $2.30 \times 10^{-5} \mathrm{~cm}^{2} / \mathrm{s}$ and the experimental reorientation time ${ }^{39,40}$ is $2.6 \mathrm{ps}$; the agreement of the water models with these measured values has been examined in detail previously. ${ }^{17}$ ) In both cases, the effective rate constants, i.e., $D$ and $1 / \tau_{0}$ as well as $1 / \tau_{2}$ and $1 / \tau_{0}$, are nearly perfectly linearly related, consistent with the assumption that the diffusive and reorientation dynamics are determined by H-bond jumps. However, it is interesting to note that for the $\mathrm{OH}$ reorientation time, Fig. 1b, the slope of $1 / \tau_{2}$ versus $1 / \tau_{0}$ is significantly different than one, indicative of the differences between the two timescales ${ }^{4,5}$ that will be discussed in detail below. (Naturally, a similar correspondence cannot be explored for $D$ given the different units for the two timescales.) 

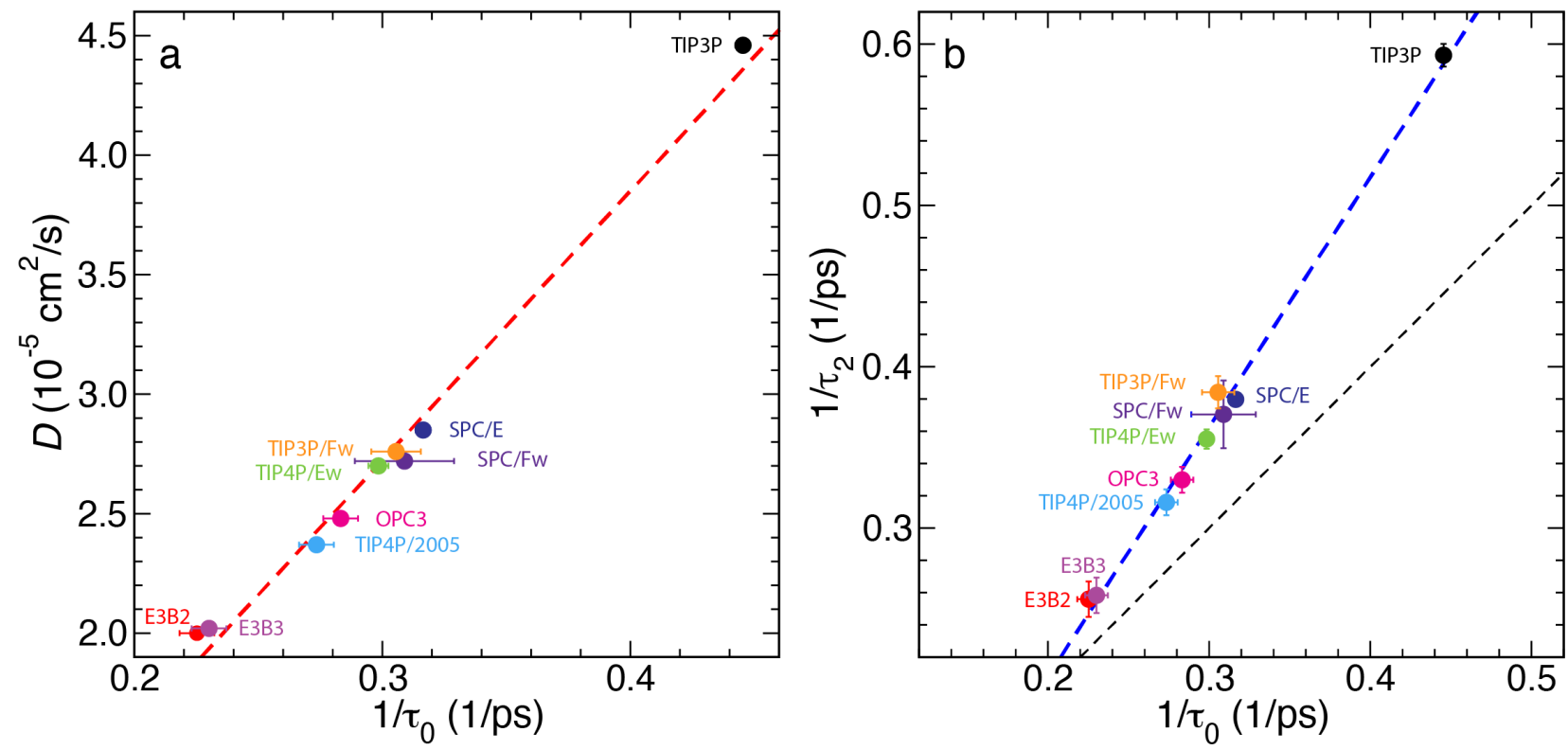

Figure 1: a) Diffusion coefficient, $D$, and b) inverse $\mathrm{OH}$ reorientation time, $1 / \tau_{2}$, for each of nine different water models plotted as a function of the inverse H-bond jump time, $1 / \tau_{0}$. Results are for $T=298.15 \mathrm{~K}$ and 1 bar. A linear fit to the data is also shown (red or blue dashed line) and, in b), a one-to-one correlation (dashed black line). Data taken from Ref. 17.

By themselves, the results in Fig. 1 illustrate only an empirical correlation between key measurable, dynamical timescales of liquid water and the H-bond jump time. (It will be interesting in the future to examine whether this correlation holds for polarizable and ab initio water models.) As noted, there is a rigorous theoretical framework, the extended jump model $(\mathrm{EJM}),{ }^{4,5}$ that connects the $\mathrm{OH}$ reorientation time, $\tau_{2}$, to the jump time and we will examine this relationship in detail below in Sec. 3. An analogous framework relating the diffusion coefficient to $\mathrm{H}$-bond exchanges is currently under development. Neither, however, predicts the relationships shown in Fig. 1. These models suggest that $D$ and $\tau_{2}$ arise from a sum of contributions coming respectively from the jump time $\tau_{0}$, from the translational or rotational jump amplitude, and from more collective structural dynamics. Further work is required to determine why $D$ and $1 / \tau_{2}$ exhibit such linear dependence with $1 / \tau_{0}$ and to probe the broader generality of these linear relationships and their causal underpinnings.

However, the latter can be partially explored, as anticipated above, by examination of the cor- responding activation energies. The diffusion coefficient and $\mathrm{OH}$ reorientation time activation energies are each plotted as a function of the jump time activation energy in Fig. 2 for the same set of water models. While all three timescales involved are non-Arrhenius, ${ }^{3,13,41,42}$ i.e., their activation energy defined by eq. 3 changes significantly with temperature, fluctuation theory for dynamics gives the activation energy at a single temperature without the necessity of choosing a temperature range in an Arrhenius plot, minimizing this complication. Fig. 2 shows that the activation energies for diffusion and reorientation are strongly, linearly correlated with that of the H-bond jump time. Both $E_{a, D}$ and $E_{a, 2}$ are larger in magnitude than $E_{a, 0}$ indicating that the additional factors influencing the motion, i.e., the size of the angular/translational jumps and the motion of the water molecule in intact $\mathrm{H}$-bonds, add to the effective barriers. Despite this, the linear correlations of $E_{a, D}$ and $E_{a, 2}$ with the jump time activation energy have slopes that are nearly unity (1.02 and 1.18, respectively). This suggests that these additional contributions to the diffusive and reorientational activation energies 

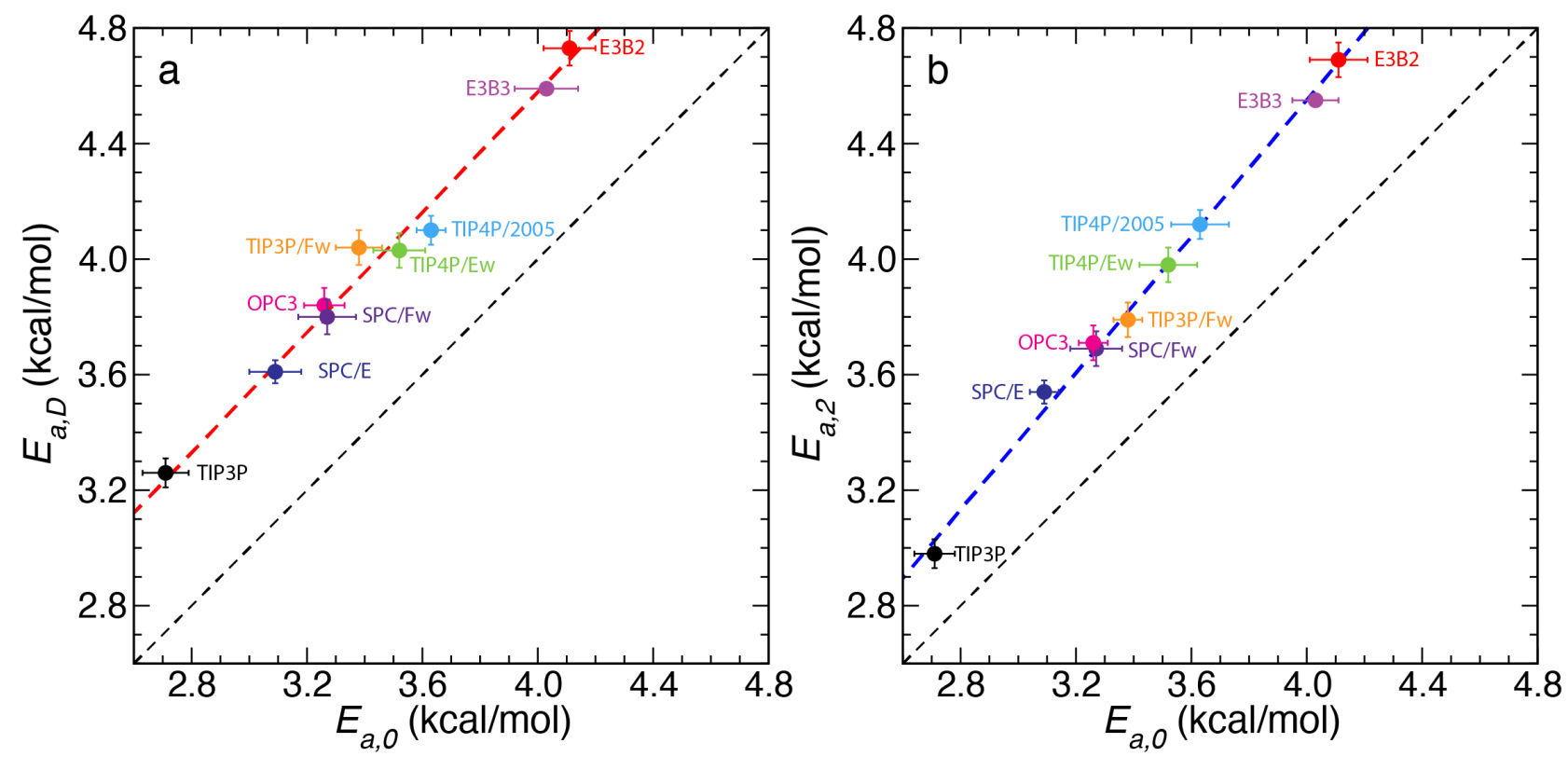

Figure 2: Jump activation energy, $E_{a, 0}$, for each of nine different water models plotted as a function of a) the water diffusion activation energy, $E_{a, D}$, and b) the $\mathrm{OH}$ reorientation activation energy, $E_{a, 2}$. Results are for $T=298.15 \mathrm{~K}$ and 1 bar. A linear fit to the model results is also shown (red or blue dashed line) in each case along with a one-to-one correlation (dashed black line). Data taken from Ref. 17.

are themselves independent of $E_{a, 0}$ or linearly, but weakly, correlated to it. Naively, one might also infer that they are quantitatively small, but we will see below that the situation, at least for $\mathrm{OH}$ reorientation, is more complicated.

Empirical correlations like those in Figs. 1 and 2 are potentially useful if they are, in fact, general. Namely, the diffusion coefficient and $\mathrm{OH}$ reorientation time can be predicted to within $\sim 6 \%$ from a calculation of the $\mathrm{H}$ bond jump time (a similar correlation allows the viscosity, not shown, to be similarly determined within $\sim 10 \%$ ). The activation energies for $D$ and $\tau_{2}$ are predicted even more accurately, to within $3 \%$ across the nine water models, from the linear correlations. Measured diffusion activation energies fall in the range $E_{a, D}=4.2-4.6 \mathrm{kcal} / \mathrm{mol},{ }^{14,43-46}$ which would predict, from Fig. 2 the jump activation energy as $E_{a, 0}=3.6-4.0 \mathrm{kcal} / \mathrm{mol}$; these values overlap with analogous predictions from measured $\mathrm{OH}$ reorientation times, ${ }^{39,40}$ but the values of $E_{a, 2}=3.7 \pm 0.5$ and $4.1 \pm 0.5 \mathrm{kcal} / \mathrm{mol}$ are sufficiently uncertain as to make the comparison of limited value.

\subsection{Mechanistic Insight from Ac- tivation Energy Decomposi- tions}

The causal link between the dynamical properties of water and H-bond exchanges can be further explored through the fluctuation theory for dynamics approach. While other approaches to determine activation energies are available, a key advantage of this theory is that it provides otherwise unavailable insight into the origin of the activation energy through a rigorous decomposition. Specifically, the fluctuation in the total system energy in the expression for the activation energy, eq. 9, can be expressed as a sum of components; for example, in classical molecular dynamics simulations a natural dissection is $\Delta H(0)=\delta K E(0)+\delta V_{L J}(0)+\delta V_{\text {Elec }}(0)$, where the three terms on the right-hand-side are the fluctuation in kinetic energy, Lennard-Jones potential energy, and electrostatic interactions, respectively. Substitution of this expression into eq. 9 gives

$$
E_{a, D}=E_{a, D, K E}+E_{a, D, L J}+E_{a, D, E l e c}
$$


where, e.g.,

$$
E_{a, D, L J}=\frac{\lim _{t \rightarrow \infty}\left\langle\delta V_{L J}(0)|\vec{r}(t)-\vec{r}(0)|^{2}\right\rangle}{\lim _{t \rightarrow \infty}\left\langle|\vec{r}(t)-\vec{r}(0)|^{2}\right\rangle} .
$$

The Tolman viewpoint for the activation energy gives a clear interpretation of this component as the average Lennard-Jones potential energy of diffusing species minus the average Lennard-Jones potential energy of all species. The $E_{a, D, K E}$ and $E_{a, D, E l e c}$ are given by corresponding expressions, with analogous interpretations.

It is then informative to compare the activation energy components for different dynamical properties of water. This is done in Fig. 3, which shows the total activation energy and its electrostatic, Lennard-Jones, and kinetic energy components for the diffusion coefficient, $\mathrm{OH}$ reorientation time, $\mathrm{H}$-bond exchange ("jump") time, and shear viscosity. (The shear viscosity is obtained from a time correlation function involving the elements of the pressure tensor; the calculation of its activation energy has been described elsewhere. ${ }^{22}$ ) The total activation energy for these $\mathrm{H}$-bond jumps are quite similar to those for diffusion and $\mathrm{OH}$ reorientation as well as the water shear viscosity.

More revealing, however, is that the energetic components of the activation energy are also quantitatively comparable for all four dynamical properties. Indeed, the individual kinetic energy, Lennard-Jones, and electrostatic components of the diffusion and $\mathrm{OH}$ reorientation activation energies are nearly identical. This is consistent with the suggestion of a common mechanism for the two dynamical timescales, namely the exchange of $\mathrm{H}$-bond partners that is the elementary step of water structure rearrangements (see also below). Yet, there are quantitative differences between the $\mathrm{H}$-bond jump time activation energy components and those of the other dynamical properties, indicative of additional features - beyond H-bond exchanges - of the mechanisms of diffusion, reorientation and viscosity that add to the energetic barriers.

Of particular note is the fact that $E_{a, L J}$ is

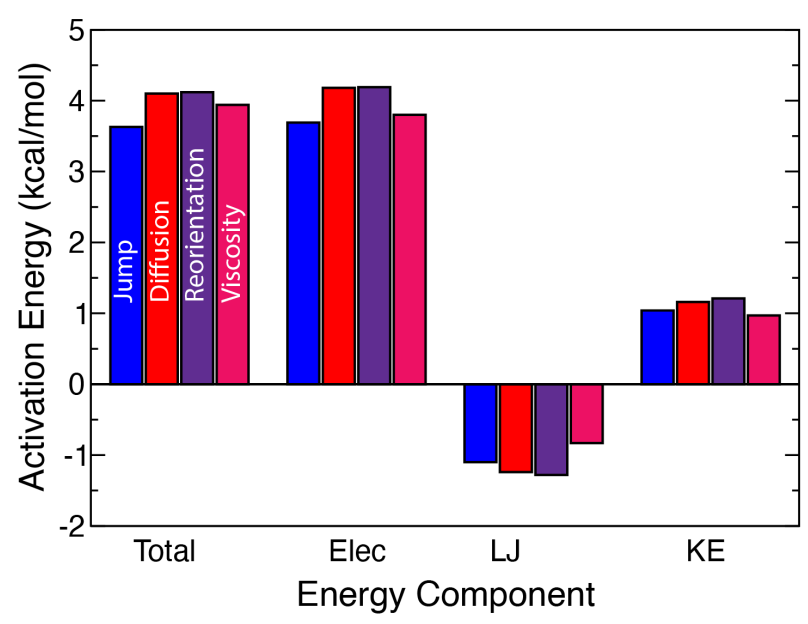

Figure 3: Total activation energy and its electrostatic, Lennard-Jones, and kinetic energy components for the H-bond jump time (blue), diffusion coefficient (red), reorientation time (purple), and shear viscosity (magenta) of TIP4P/2005 water. Data taken from Refs. 17 and 22 .

negative for each of these measures of water dynamics. Within the Tolman perspective, this indicates that the water dynamics are faster when the Lennard-Jones interaction energy is smaller. We have previously noted that this results from a competition inherent in the $\mathrm{H}$ bond, which is held together by electrostatic interactions (within the classical models used in these simulations) that are restrained by the repulsive Lennard-Jones interactions of the two partnered water molecules. In breaking this H-bond, the electrostatic energy rises but the Lennard-Jones energy falls as the two molecules separate; see Fig. 5 in Ref. 19.

\section{A Rigorous Connection: The Extended Jump Model for $\mathrm{OH}$ Reorien- tation in Water}

The connection between the $\mathrm{OH}$ reorientation in water and H-bond exchanges has been formalized in the extended jump model (EJM) developed by Laage and Hynes. ${ }^{4,5}$ In the EJM, the $\mathrm{OH}$ reorientation time is expressed as a sum of 
contributions from $\mathrm{H}$-bond jumps between acceptors that occur with a large change in the $\mathrm{OH}$ angle and the "frame" reorientation of the intact H-bonded pair,

$$
\frac{1}{\tau_{2}}=\frac{1}{\tau_{2, \text { jump }}}+\frac{1}{\tau_{2, \text { frame }}} .
$$

Here, $\tau_{2, \text { frame }}$ is the longest decay timescale of the second-order reorientational time correlation function $(\mathrm{TCF})$ for the $\mathrm{OO}$ bond in the intact H-bond $C_{2, \text { frame }}(t)=\left\langle P_{2}\left[\vec{e}_{O O}(0)\right.\right.$. $\left.\left.\vec{e}_{O O}(t)\right]\right\rangle_{H-b o n d}$. The timescale $\tau_{2, \text { jump }}$ is the contribution from jumps between H-bond acceptors based on the jump time, $\tau_{0}$ (the inverse of the rate constant for the "reaction" of an $\mathrm{H}$ bond exchange), and the size of the associated large angle changes,

$$
\tau_{2, \text { jump }}=\frac{\tau_{0}}{\bar{\omega}_{2}},
$$

where $\bar{\omega}_{2}$ is the average value of the weighting in the $C_{2}(t)$ TCF for angular jump size, $\Delta \theta$,

$$
\omega_{2}(\Delta \theta)=\left\{1-\frac{\sin [5 \Delta \theta / 2]}{5 \sin [\Delta \theta / 2]}\right\} .
$$

These equations defining the EJM give a direct relationship between the jump and reorientation times and the associated activation energies.

The $\tau_{2}$ reorientation time activation energy is given by a weighted combination of the activation energies for the jump and frame components, ${ }^{19}$

$$
E_{a, 2}=\frac{\tau_{2}}{\tau_{2, \text { jump }}} E_{a, 2}^{\text {jump }}+\frac{\tau_{2}}{\tau_{2, \text { frame }}} E_{a, 2}^{\text {frame }} .
$$

The jump contribution activation energy is itself a combination of the activation energy associated with the jump time, $\tau_{0}$, and the weight $\bar{\omega}_{2}$ that depends on the angles traversed when $\mathrm{H}$-bonds are exchanged,

$$
\begin{aligned}
E_{a, 2}^{\text {jump }} & =\frac{1}{\tau_{0}} \frac{\partial \tau_{0}}{\partial \beta}-\frac{1}{\bar{\omega}_{2}} \frac{\partial \bar{\omega}_{2}}{\partial \beta} \\
& =E_{a, 0}+E_{a, 2}^{\Delta \theta} .
\end{aligned}
$$

Thus, we see that the $\mathrm{OH}$ reorientational activation energy that is obtained in an infrared pump-probe anisotropy measurement is related to three contributions,

$$
E_{a, 2}=\frac{\tau_{2}}{\tau_{2, \text { jump }}}\left(E_{a, 0}+E_{a, 2}^{\Delta \theta}\right)+\frac{\tau_{2}}{\tau_{2, \text { frame }}} E_{a, 2}^{\text {frame }},
$$

only one of which is the H-bond jump activation energy, $E_{a, 0}$. The activation energy predicted by the model was shown ${ }^{19}$ to be in excellent agreement with that calculated directly from simulations.

The EJM result in eq. 17 shows the direct connection between the H-bond exchange activation energy, $E_{a, 0}$, and the $\mathrm{OH}$ reorientation time activation energy, $E_{a, 2}$. However, it also makes clear that the two are not the same, with $E_{a, 2}$ having additional contributions from the reorientation of the intact $\mathrm{H}$-bonded pair and the temperature-dependence of the angular jumps that occur upon $\mathrm{H}$-bond exchange. This is illustrated in Fig. 4. It will be interesting to examine this behavior in linear alcohols where, for some chain lengths, the frame time is faster than the H-bond jump time. ${ }^{47}$ Similar behavior is expected for the relation between the jump and diffusion coefficient, $E_{a, D}$, activation energies, which must involve how the distance moved by a water molecule undergoing $\mathrm{H}$-bond exchange and diffusion of a persistently $\mathrm{H}$-bonded water molecule depend on temperature.

Despite these confounding factors, $E_{a, 2}$ and $E_{a, 0}$ are strongly, linearly correlated and so are $E_{a, D}$ and $E_{a, 0}$, as shown in Fig. 2 for nine different water models. In a detailed analysis of the EJM for the SPC/E water model, ${ }^{17}$ we found that $E_{a, 2}^{\Delta \theta}$ is comparatively small ( $0.13 \mathrm{kcal} / \mathrm{mol})$, while $E_{a, 2}^{\text {frame }}$ is substantial, $3.66 \mathrm{kcal} / \mathrm{mol}$, but reasonably similar to $E_{a, 0}=$ $3.31 \mathrm{kcal} / \mathrm{mol}$. This is perhaps not surprising in that the frame reorientation of the intact $\mathrm{H}$ bonded pair itself is presumed to occur by the $\mathrm{H}$-bond exchanges of water molecules solvating the pair, leading to a comparable activation energy. In other words, because liquid water is fundamentally defined by its H-bond network, nearly any rearrangement involves the exchange of H-bonds and hence has a similar activation energy. However, lest one become too comfort- 


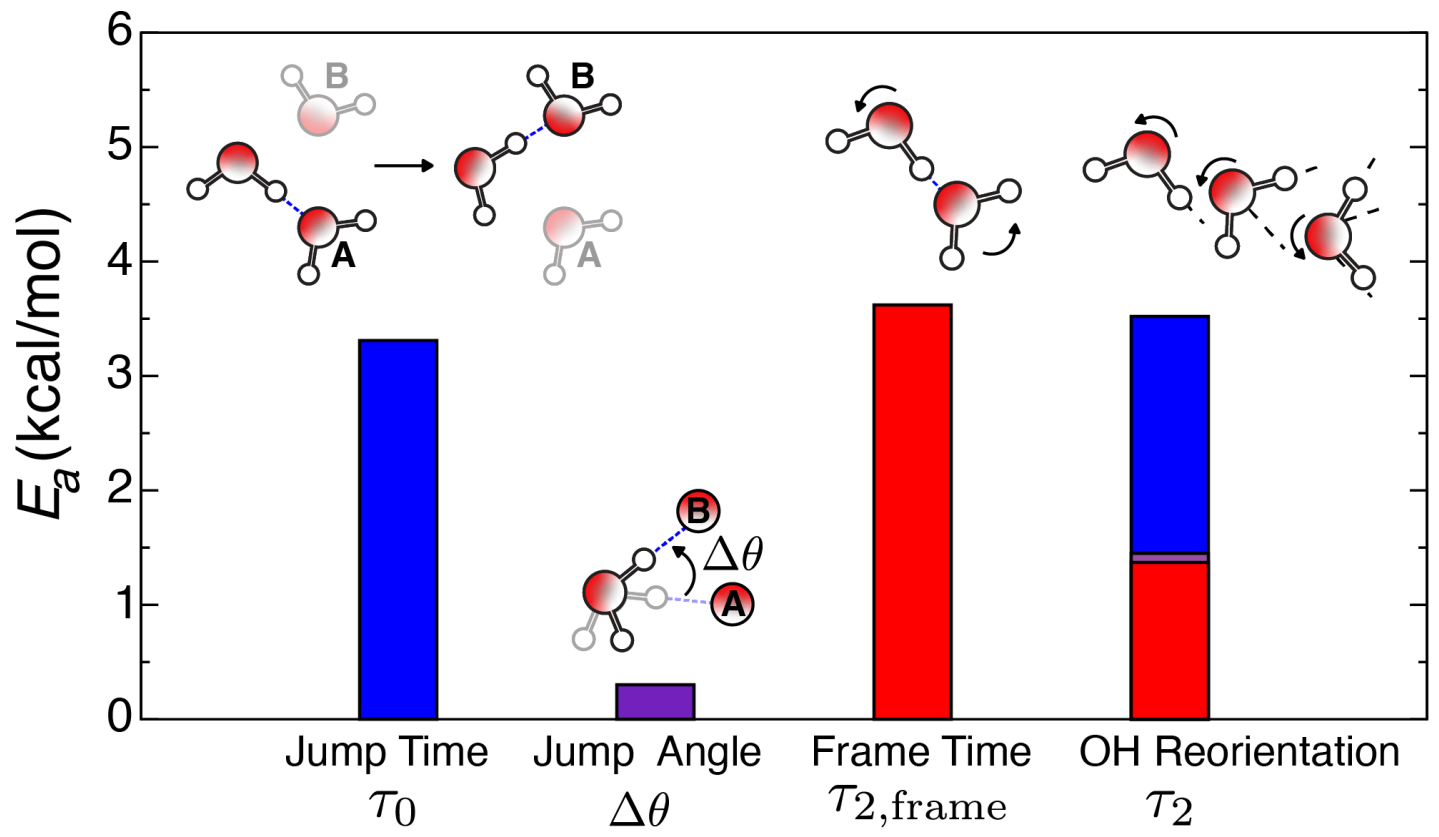

Figure 4: Illustration of the contributions to the $\mathrm{OH}$ reorientation time, $\tau_{2}$, activation energy (rightmost bar) given by eq. 17. The activation energies associated with the H-bond jump time, $\tau_{0}$, (blue), the contribution from the size of the angular traversed, $\Delta \theta$, during a jump (purple), and the frame reorientation time, $\tau_{2, \text { frame }}$, of the intact $\mathrm{H}$-bonded pair (red) are shown along with schematic figures of the associated motion. Data are for the SPCE water model ${ }^{30}$ and taken from Ref. 19.

able with such a painless description of water dynamics, a key counterexample is presented in the next Section.

\section{A Counterexample: Vi- brational Spectral Diffu- sion in Water}

While the analysis of activation energies provides strong support for a common structural rearrangement origin for translational and reorientational dynamics in water, we now show that it can also reveal that other dynamical processes taking place on a similar picosecond (ps) timescale are not caused by the same underlying mechanism. We now illustrate this point with a different dynamical property of water, the spectral diffusion time, which has attracted significant attention over the past two decades, motivated by two-dimensional infrared spectroscopy (2D-IR) measurements. The spectral diffusion time is a measure of the dynamics of the $\mathrm{OH}$ (or OD) vibrational frequency obtained from the decay of the frequency-frequency time correlation function (FFCF),

$$
C_{\omega}(t)=\left\langle\delta \omega_{01}(0) \delta \omega_{01}(t)\right\rangle .
$$

Here, $\delta \omega_{01}(t)=\omega_{01}(t)-\left\langle\omega_{01}\right\rangle$ where $\omega_{01}(t)$ is the fundamental vibrational frequency of the $\mathrm{OH}$ mode. The FFCF can be obtained from the center-line-slope of the $2 \mathrm{D}$-IR spectrum ${ }^{48}$ and thus is directly measurable.

In liquid water at ambient conditions, $\mathrm{OH}$ spectral dephasing occurs on a ps timescale, typically associated with $\mathrm{H}$-bond rearrangements. Comparison between simulations and measurements lead to the proposal by Lawrence and Skinner that the longer-time decay of the FFCF directly probes $\mathrm{H}$-bond exchange dynamics. ${ }^{49}$ They compared the FFCF to time correlation functions that measure continuous survival probability of H-bonds and found that they decay on the same time scale.

It is noteworthy that the H-bond TCF used by Lawrence and Skinner are not the same as 
that which provides the H-bond jump time. ${ }^{4,5}$ The jump time is obtained from the time constant of the exponential decay of the stablestates TCF, ${ }^{4,5} 1-C_{A B}(t)$ where $C_{A B}(t)=$ $\left\langle n_{A}(0) n_{B}(t)\right\rangle$ with $n_{A}=1(0)$ when an $\mathrm{OH}$ is (is not) H-bonded to acceptor $A$ and $n_{B}=1(0)$ when it is (is not) $\mathrm{H}$-bonded to a new acceptor $B$. The principal difference is that they decay on timescales faster than the jump time because they are sensitive to transient H-bond breaks, i.e., $\mathrm{OH}$ groups that briefly break their H-bond but do not find a new acceptor and instead return to the original acceptor. This deviation between the spectral diffusion time and the jump time was resolved by Lawrence and Skinner by describing the former as arising from the sum of the forward and backward jump rate constants for a given $\mathrm{OH}$.

Such plausible arguments about the connection between the spectral diffusion and jump timescales are directly tested by examination of the corresponding activation energies. ${ }^{26} \mathrm{We}$ have recently calculated the spectral diffusion and jump activation energies, $E_{a, \omega}$ and $E_{a, 0}$, respectively, for three water models. The results are presented in Fig. 5 and show that the spectral diffusion time has a significantly lower activation energy than the jump time. Not only does this demonstrate that the two timescales measure different dynamics, it indicates that $\mathrm{H}$ bond jumps require passage over higher barriers and hence spectral diffusion does not require H-bond exchange. We did find a small $(<3 \%$ amplitude) component in the FFCF (see the right panel of Fig. 5) that has a timescale and activation energy matching the jump time; this amplitude is likely not large enough, or at short enough times, to appear in measurements of the FFCF and spectral diffusion time.

Analyzing their respective activation energies therefore reveals that $\mathrm{OH}$ frequency dephasing is not sensitive to the same H-bond network rearrangements as translational and rotational dynamics: while unsuccessful attempts at jump H-bond exchanges do not lead to stable water reorientation and translation, they do induce $\mathrm{OH}$ frequency dephasing.

\section{An Open Challenge: Non-Arrhenius Behavior}

The above examples show how activation energies provide important insight into the connection between $\mathrm{OH}$ reorientation dynamics and $\mathrm{H}$ bond jumps as well as the lack of a connection between spectral diffusion and H-bond jumps. However, a key feature of water dynamics is that they are non-Arrhenius, i.e., the activation energy itself depends on temperature.

This non-Arrhenius behavior is often connected to the existence of a liquid-liquid phase transition for water that is now generally accepted. ${ }^{21,50-53}$ The phase coexistence line terminates at a critical point around 1700 bar and its extension to lower pressures, called the Widom line, is believed to lie around $215 \mathrm{~K}$ at 1 bar. ${ }^{50-52}$ This leads to a continuous change in the water structure from predominantly high density liquid (HDL) at temperatures above this point to mostly low density liquid (LDL) below this point, the so-called "two liquids" picture. The LDL structure is more ordered, with greater tetrahedrality in the water first solvation shell. This has significant consequences for water dynamics such as diffusion and reorientation. ${ }^{21,50,53,54}$ This is illustrated, over a limited temperature range of 235-360 K, in Fig. 6 based on 1 bar constant pressure simulations with the TIP4P/2005 water model.

This behavior is described by the temperature dependence of the activation energy, ${ }^{16,21}$ namely

$$
\begin{aligned}
\frac{\partial E_{a, D}}{\partial \beta} & =-\frac{\lim _{t \rightarrow \infty}\left\langle\left[\delta H(0)^{2}-\left\langle\delta H^{2}\right\rangle\right]|\vec{r}(t)-\vec{r}(0)|^{2}\right\rangle}{\lim _{t \rightarrow \infty}\left\langle|\vec{r}(t)-\vec{r}(0)|^{2}\right\rangle} \\
& +E_{a, D}^{2}
\end{aligned}
$$

The non-Arrhenius behavior of water dynamics can be interpreted, within some assumptions, in terms of eq. 1. Imagining a non-recrossing dividing surface, we have (under constant pressure conditions),

$$
\ln k(T)=\ln \nu_{s}-\beta \Delta H^{\ddagger}+\frac{\Delta S^{\ddagger}}{k_{B}},
$$




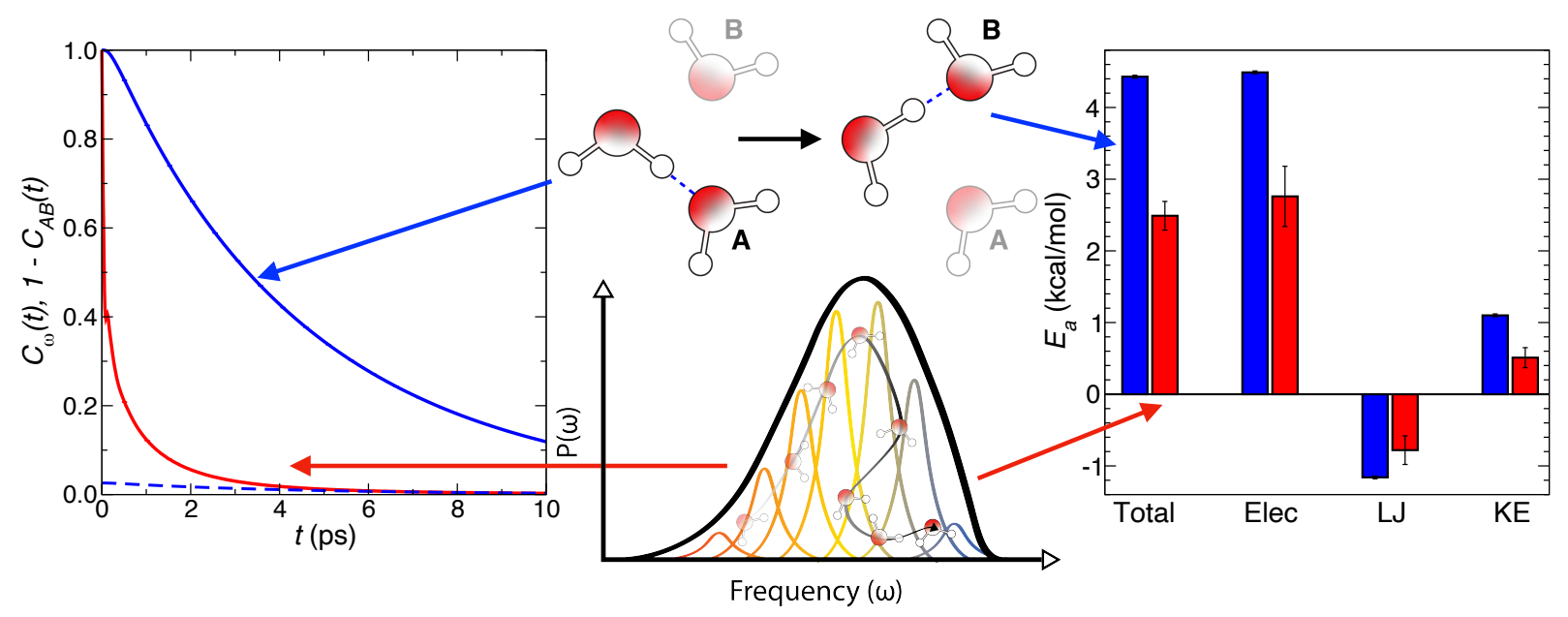

Figure 5: Comparison of H-bond jump and spectral diffusion dynamics, schematically illustrated in the central panel. Their respective time correlation functions, the stable states TCF, $1-C_{A B}(t)$, that decays with a time constant of $\tau_{0}$ (solid blue line) and the FFCF, $C_{\omega}(t)$, (solid red line) are compared in the left panel; the jump time contribution to the FFCF is also shown (dashed blue line). The total activation energies for the jump time (blue) and spectral diffusion (red) and their energetic contributions are shown in the right panel. Data are for the E3B2 water model ${ }^{37}$ and taken from Ref. 26.

and then with eq. 3, we obtain $E_{a}=\Delta H^{\ddagger}$. The typical van't Hoff/Arrhenius assumption is that $\Delta H^{\ddagger}$ and $\Delta S^{\ddagger}$, the activation enthalpy and entropy, respectively, are temperature independent, which is clearly not the case for water dynamics. In this context for the case of water diffusion,

$$
\frac{\partial E_{a, D}}{\partial \beta}=\frac{\partial \Delta H^{\ddagger}}{\partial \beta},
$$

can be understood as a measure of the temperature dependence of $\Delta H^{\ddagger}$ and thus of the change in the underlying barrier for diffusion, associated with the changes noted above in the water structure, as the temperature is changed.

This measure of the non-Arrhenius character of the dynamics can be used to accurately describe the behavior of timescales over larger temperature ranges. A key example is the selfdiffusion coefficient of water that exhibits a qualitative change moving across the Widom line at 1 bar by supercooling. ${ }^{52-54}$ This has been measured in thin layers of water by Kimmel and co-workers ${ }^{54}$ and it has been suggested that the data can be quantitatively described as, ${ }^{52}$

$$
\begin{aligned}
\ln D(T) & =s(T) \ln D_{L D L}(T) \\
& +[1-s(T)] \ln D_{H D L}(T)
\end{aligned}
$$

where $D_{L D L}(T)=D_{0, L D L} e^{-\beta E_{a, L D L}}$ and $D_{H D L}(T)=D_{0, H D L} e^{-\beta E_{a, H D L}}$ are Arrhenius descriptions of the low- and high-density liquids, respectively, and $s(T)=1 /[1+$ $\left.e^{4.394\left(T-T_{0}\right) / \Delta T}\right]$ is a switching function centered around the Widom line temperature. We have fit the six parameters $\left(D_{0, L D L}, E_{a, L D L}, D_{0, H D L}\right.$, $\left.E_{a, H D L}, T_{0}, \Delta T\right)$ in eq. 22 to the values of $D$, $E_{a, D}$, and $\partial E_{a, D} / \partial \beta$ obtained in simulations of TIP4P/2005 water at room temperature alone. The fit parameters depend somewhat on the initial values assumed, but the most frequently obtained values are used to calculate $D(T)$ from eq. 22 and compared to the experimental measurements of Ref. 54 and direct simulations of $D(T)$ in Fig. 6.

This shows that a non-Arrhenius temperature dependence does not necessarily imply a change in the underlying mechanism. In supercooled liquid water, it was shown ${ }^{42}$ that the H-bond exchange mechanism is unchanged when the temperature decreases and remains the main reorientation pathway, but that its temperature dependence is non-Arrhenius because of the progressive shift in the distribution of local structures, which causes a change in the activation energy. 

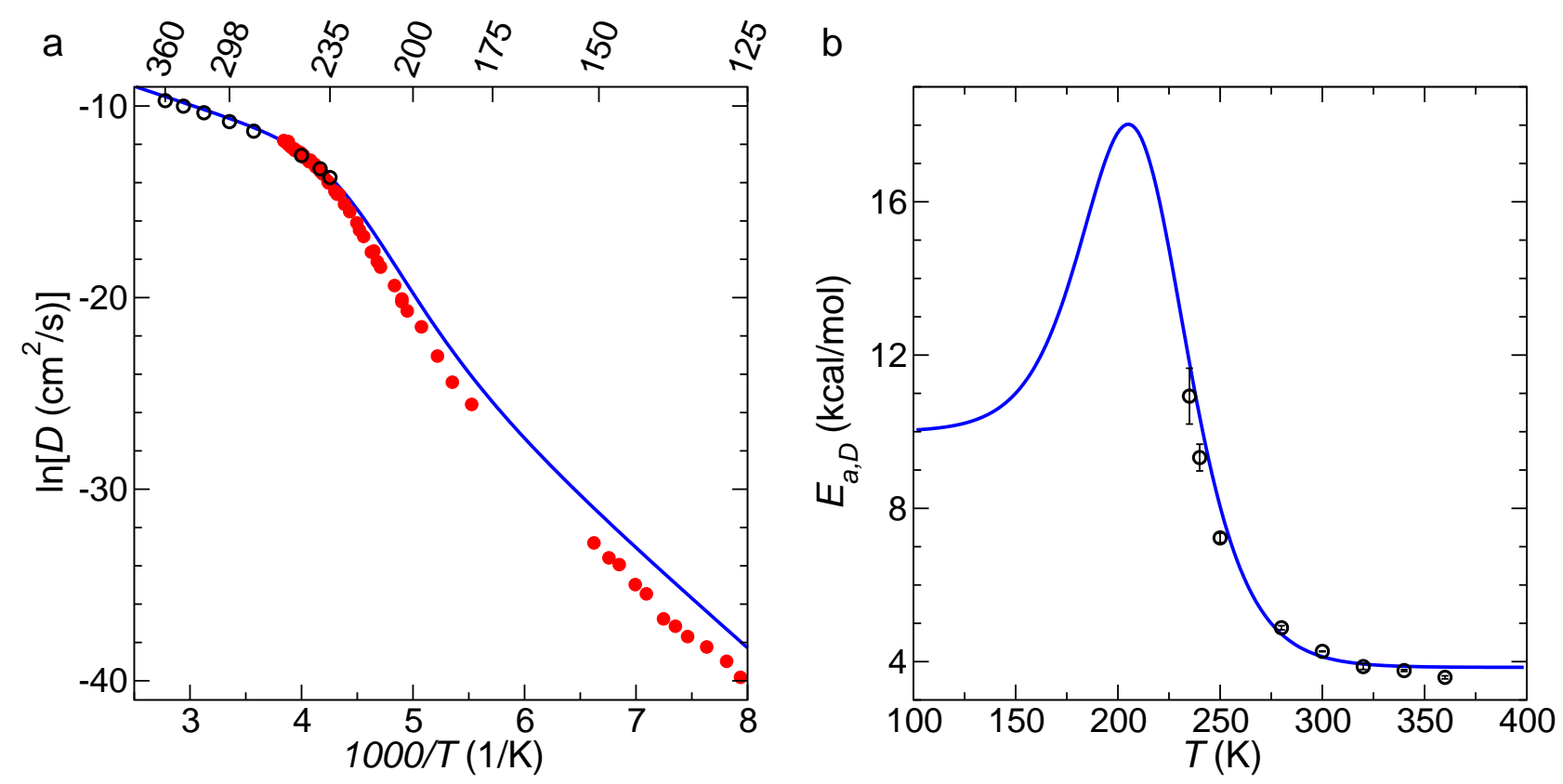

Figure 6: a) Plot of $D(T)$ at 1 bar versus $T$ from $\mathrm{Xu}$ et al. ${ }^{54}$ (red circles), direct simulations at different $T$ (black circles), and eq. 22 parameterized from $D, E_{a, D}$, and $\partial E_{a, D} / \partial \beta$ calculated at 298.15 K (blue line) for TIP4P/2005. b) Corresponding predicted (blue line) and directly simulated (black circles) $E_{a, D}$ versus $T$. Simulation results taken from Ref. 21.

\section{An Open Challenge: Ac- tivation Volumes}

The discussion above has focused exclusively on the temperature dependence of dynamical properties of water. However, the effects of other thermodynamic variables are also important and can provide additional mechanistic insight. Key among these variables is pressure, the impact of which is typically measured by the activation volume,

$$
\Delta V^{\ddagger}=-k_{B} T \frac{\partial \ln k}{\partial p},
$$

for a rate constant, $k$, or timescale, $\tau=1 / k$. This is a measure of the difference in volume occupied by the transition state for the process and the reactants; it is important to note that the relevant volume includes any changes in solvent structure. ${ }^{55-57}$

In the case of water, the pressure dependences of dynamical properties such as the diffusion coefficient and the integrated reorientation time (the integral of $C_{2}(t)$ accessible from NMR) have been examined. ${ }^{17,24,43,44,58,59}$ These prop- erties are not "Arrhenius" in pressure - i.e., $\ln k$ is not linear in $p$ so that $\Delta V^{\ddagger}$ changes significantly with pressure - and thus activation volumes are typically determined by measuring a timescale at a large number of pressures and fitting the results to a functional form. ${ }^{43,44,58,59}$ At the same time, even relatively small changes in the timescale usually require increasing the pressure by hundreds or thousands of bar, which has limited the attention of simulation studies.

The fluctuation theory for dynamics approach enables the calculation of the activation volume from simulations at a single pressure. ${ }^{17,24}$ In analogy with eq. 9, the activation volume for the diffusion coefficient is given by

$$
\Delta V_{D}^{\ddagger}=\frac{\lim _{t \rightarrow \infty}\left\langle\delta V(0)|\vec{r}(t)-\vec{r}(0)|^{2}\right\rangle_{N p T}}{\lim _{t \rightarrow \infty}\left\langle|\vec{r}(t)-\vec{r}(0)|^{2}\right\rangle_{N p T}},
$$

where $\delta V(0)=V(0)-\langle V\rangle$ is the fluctuation at $t=0$ in the total system volume and the subscript $N p T$ indicates an average computed in the isothermal-isobaric ensemble.

We have previously calculated $\Delta V_{D}^{\ddagger}$ using eq. 24 at several different temperatures and 

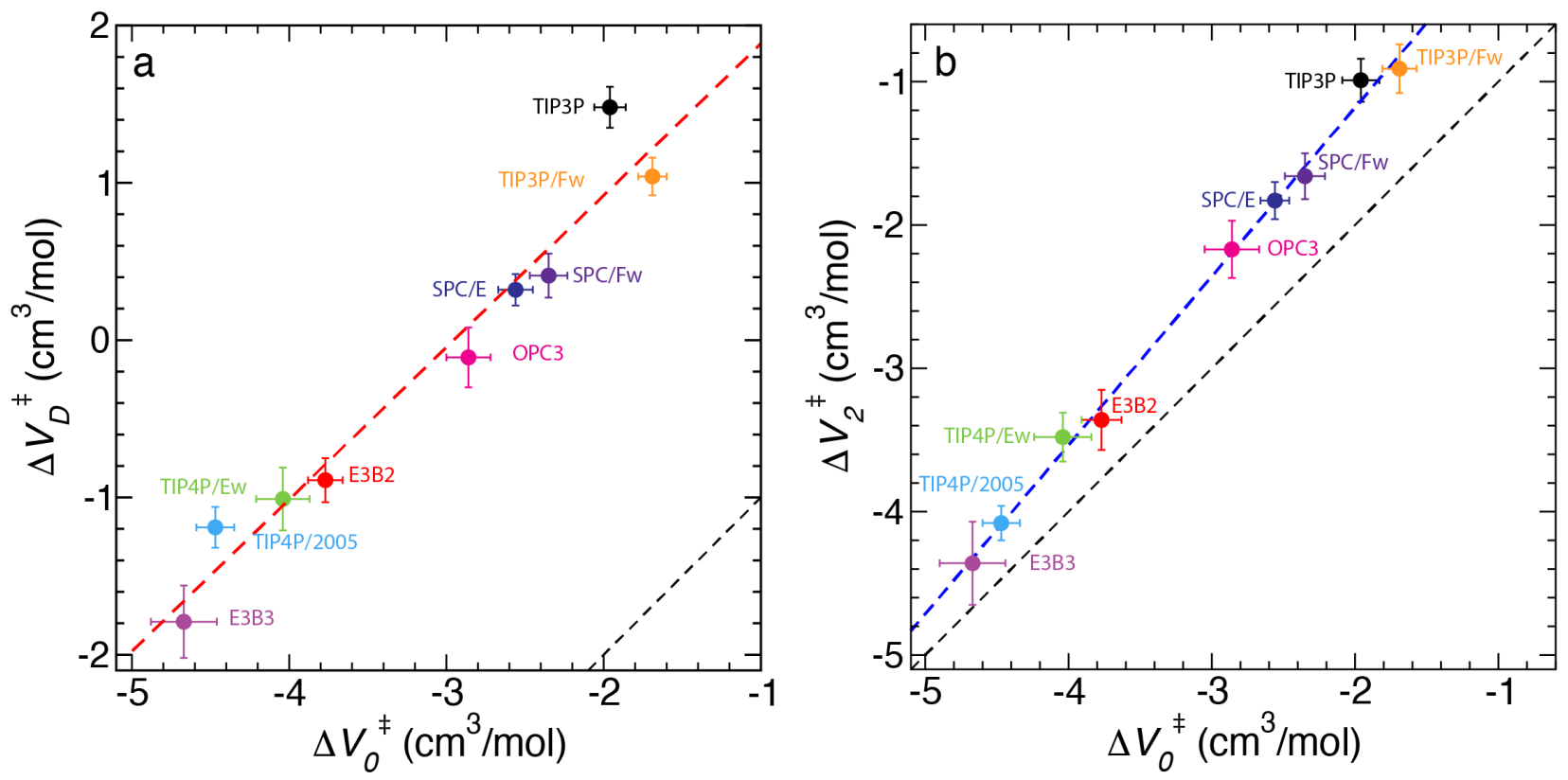

Figure 7: The activation volume for a) the diffusion coefficient, $\Delta V_{D}^{\ddagger}$, and b) the $\mathrm{OH}$ reorientation time, $\Delta V_{2}^{\ddagger}$, are plotted against the H-bond jump time activation volume, $\Delta V_{0}^{\ddagger}$, for each of nine water models. A linear fit to the model results is also shown (red or blue dashed line) in each case along with a one-to-one correlation (dashed black line). Data taken from Ref. 17.

pressures for $\mathrm{SPC} / \mathrm{E}$ water. ${ }^{24}$ The results are in good agreement with previous experimental measurements. ${ }^{43,44,58,59}$ More recently we have computed the activation volumes for the diffusion coefficient, $\mathrm{OH}$ reorientation time $\left(\tau_{2}\right)$, and the H-bond jump time $\left(\tau_{0}\right)$ for several water models. ${ }^{17}$

It is then interesting to compare these in the same manner as we have done for activation energies in Fig. 2. This is presented in Fig. 7 where $\Delta V_{D}^{\ddagger}$ and $\Delta V_{2}^{\ddagger}$ are each plotted as a function of the H-bond jump activation volume, $\Delta V_{0}^{\ddagger}$. The results bear some similarities to the activation energies in Fig. 2 in that the activation volumes are all strongly, linearly correlated. The slope of this correlation is nearly unity (0.96) for the case of the diffusion coefficient, but somewhat higher (1.18) for the reorientation time. This is contrasted, however, by a significant shift in the diffusion coefficient activation volume relative to that for $\mathrm{H}$-bond jumps. Namely, $\Delta V_{D}^{\ddagger}$ is systematically higher than $\Delta V_{0}^{\ddagger}$ by $\sim 3 \mathrm{~cm}^{3} / \mathrm{mol}$. Note that a comparatively smaller, $\sim 0.55 \mathrm{kcal} / \mathrm{mol}$, analogous shift is observed for the diffusion coefficient activation energies relative to those for the $\mathrm{H}$ - bond jump time.

Taken together, these differences between the activation parameters for diffusion and the jump time indicate important components of the water diffusion mechanism beyond the exchange of H-bond partners. (It is noteworthy that such mechanistic insight is not derivable from examination of the associated timescales alone.) In particular, the larger values of $\Delta V_{D}^{\ddagger}$ compared to the quite negative jump time activation volumes indicate that water diffusion involves components, e.g., the translational motion upon $\mathrm{H}$-bond exchange, that are significantly more sensitive to the available volume than the H-bond jump itself. Similarly, the larger $E_{a, D}$ relative to $E_{a, 0}$ show that water diffusion also requires surpassing an additional barrier beyond that of an H-bond jump. The general picture that is anticipated for diffusion is of a translational displacement associated with an H-bond exchange and diffusion of an intact H-bonded molecule; both of these components would be expected to lead to a larger activation volume and activation energy than an H-bond jump alone. The fact that $E_{a, D}$ versus $E_{a, 0}$ and $\Delta V_{D}^{\ddagger}$ versus $\Delta V_{0}^{\ddagger}$ both have slopes 
that are nearly one suggests that these additional factors have activation parameters that are relatively independent of $E_{a, 0}$ and $\Delta V_{0}^{\ddagger}$, or depend on them in opposite manners that effectively cancel. This suggests some inferences about the (lack of) a relationship between the mechanistic details of these components of diffusion and $\mathrm{H}$-bond exchanges.

Interestingly, the picture is different for the case of the $\mathrm{OH}$ reorientation time. While both the activation volume and energy are higher for $\tau_{2}$ than the jump time, the differences are smaller. In particular, the reorientation and jump time activation energies, Fig. 2b, are within $20 \%$ of each other for all the water models, and the corresponding activation volumes differ by less than $1 \mathrm{~cm}^{3} / \mathrm{mol}$. At the same time, the differences grow with the jump time activation energy or volume. This indicates that, in contrast to diffusion, the additional components of the $\mathrm{OH}$ reorientation time, specifically the magnitude of the associated angular jump and the frame reorientation time (see, e.g., eq. 17), have activation energies and volumes that scale with those of the jump time. These results can be easily rationalized as the angular jumps should be less sensitive to pressure than translational ones required for diffusion.

\section{Summary \& Future Di- rections}

In this Perspective, we have illustrated how careful consideration of activation energies reveals both the common and distinct mechanistic origins of various timescales associated with the dynamics of liquid water. At the center of water structure and dynamics is, naturally, the H-bond network and thus, not surprisingly, the exchange of $\mathrm{H}$-bond partners within this network represents the shared rate-limiting step for many (but not all!) timescales in water.

The extended jump model ${ }^{4,5}$ rigorously connects $\mathrm{H}$-bond exchanges with $\mathrm{OH}$ reorientation at both the timescale and activation energy level, as discussed in Sec. 3. An analysis of the reorientation time activation energy, ${ }^{19}$ however, shows that only $\sim 60 \%$ is associated with $\mathrm{H}$ - bond jumps and a significant contribution arises from the (slower) reorientation of the intact H-bonded pair. An analogous description for diffusion is currently being developed, which, given the strong correlation between diffusion and reorientation, can be expected to provide a similarly rigorous link and, perhaps, additional unexpected results. It remains, however, to extend these pictures to connections between $\mathrm{H}$ bond exchanges and more collective measures of water dynamics such as viscosity ${ }^{8,60}$ and dielectric relaxation. Such a unified theory of water dynamics would also shed light on other related issues of considerable insight, including breakdowns of Stokes-Einstein behavior ${ }^{22}$ and the role of $\mathrm{H}$-bonding in solvation dynamics. A key caveat, however, is revealed by examination of the spectral diffusion time (Sec. 4), which shows that $\mathrm{H}$-bond exchange is not required for all water dynamical properties. ${ }^{26}$

The fluctuation theory for dynamics approach that enables much of this analysis also permits non-Arrhenius parameters to be calculated from simulations at a single temperature, ${ }^{16,21}$ as illustrated in Sec. 5. Such information provides a more global description of the temperature dependence of dynamical timescales. Moreover, it demonstrates that the dynamical properties at all temperatures are encoded in the structural and dynamical behavior at a single (arbitrary) temperature (at least for a given phase). However, this is only an initial step and several challenges remain. First, the calculation of $\partial E_{a} / \partial \beta$ is significantly more computational intensive than that of $D$ or $E_{a}$ alone. Improved methods, e.g., for accelerated sampling, are needed to make these simulations more routine. Second, the qualitative form of dynamical time correlation functions can change over the temperature range, e.g., the water $\mathrm{OH}$ reorientational correlation function $C_{2}(t)$ changes from a long-time exponential decay to a stretched exponential decay upon supercooling. This makes even the identification of a particular timescale challenging. Third, it remains to generate enough information about the temperature dependence, e.g., through calculation of even higher derivatives, to develop model-less descriptions. Fourth, better experi- 
mental determination of $E_{a}$ versus $T$ would provide greater insight and more rigorous tests of modeling.

Consideration of activation volumes (Sec. 6) reveals important gaps in our understanding of the mechanisms of water dynamics. In particular, it indicates key differences between the volume required for the other motions, beyond $\mathrm{H}$ bond exchange, involved in diffusion and $\mathrm{OH}$ reorientation. These have received little attention and remain largely unexplained beyond the rationalizations offered in Sec. 6, but represent an important benchmark for molecular theories. At the same time, additional measurements of activation volumes, e.g., for $\mathrm{OH}$ reorientation and spectral diffusion, would further test such theories as well as simulation models.

There are many potential directions for future investigations beyond those outlined in the preceding paragraphs. For example, the detailed insight into activation energies offered by the fluctuation theory for dynamics should enable greater consideration of dynamical properties in the development of force fields for molecular simulations. Moreover, while the focus here is on liquid water, corresponding examinations of activation energies and volumes for dynamics in non-aqueous liquids, ${ }^{29}$ electrolyte solutions, ${ }^{27,29}$ nanostructured materials, and biological systems, will present new challenges but also yield improved mechanistic descriptions.

\section{Acknowledgments}

This work was supported by the National Science Foundation under Grant No. CHE2102656 (W.H.T.). This material is based on the work supported by the National Science Foundation Graduate Research Fellowship under Grant Nos. 1540502 and 1451148 as well as the National Science Graduate Research Opportunities Worldwide Program (Z.A.P.).

\section{References}

(1) Eisenberg, D.; Kauzmann, W. The Structure and Properties of Water; Oxford University Press, 1969.
(2) Rahman, A.; Stillinger, F. Molecular dynamics study of liquid water. J. Chem. Phys. 1971, 55, 3336-3359.

(3) Chen, S. H.; Teixeira, J. Structure and dynamics of low-temperature water as studied by scattering techniques. Adv. Chem. Phys. 1986, 64, 1-45.

(4) Laage, D.; Hynes, J. T. A molecular jump mechanism of water reorientation. Science 2006, 311, 832-835.

(5) Laage, D.; Hynes, J. T. On the molecular mechanism of water reorientation. $J$. Phys. Chem. B 2008, 112, 14230-14242.

(6) Wynne-Jones, W. F.; Eyring, H. The absolute rate of reactions in condensed phases. J. Chem. Phys. 1935, 3, 492-502.

(7) Wigner, E. The transition state method. Trans. Faraday Soc. 1938, 34, 29-41.

(8) Glasstone, S.; K.J., L.; Eyring, H. In The Theory of Rate Processes; McGraw-Hill, Ed.; 1941.

(9) Frequently, using the second line in eq. 1, it is assumed that $\nu_{s}=k_{B} T / h$ so that a so-called Eyring plot $^{6}$ of $\ln \left[k(T) /\left(k_{B} T / h\right)\right]$ versus $1 / T$ yields the activation internal energy, $\Delta U^{\ddagger}$, (or, at constant pressure the activation enthalpy, $\left.\Delta H^{\ddagger}\right)$ from the slope and the activation entropy, $\Delta S^{\ddagger}$, from the intercept. However, this choice of a weak temperature dependence in $\nu_{s}$ only changes $\Delta U^{\ddagger}$ by $k_{B} T$; the actual temperature dependence of $\nu_{s}$ may be greater or lesser.

(10) Bakker, H. J.; Skinner, J. L. Vibrational spectroscopy as a probe of structure and dynamics in liquid water. Chem. Rev. 2010, 110, 1498-1517.

(11) Ludwig, R.; Weinhold, F.; Farrar, T. C. Experimental and theoretical determination of the temperature dependence of deuteron and oxygen quadrupole coupling constants of liquid water. J. Chem. Phys. 1995, 103, 6941-6950. 
(12) Weingartner, H. Self-diffusion in liquid water. A reassessment. Z. Phys. Chem. 1982, 132, 129-149.

(13) Qvist, J.; Schober, H.; Halle, B. Structural dynamics of supercooled water from quasielastic neutron scattering and molecular simulations. J. Chem. Phys. 2011, $134,144508$.

(14) Mills, R. Self-diffusion in normal and heavy water in the range $1-45^{\circ}$. J. Phys. Chem. 1973, 7\%, 685-688.

(15) Mesele, O. O.; Thompson, W. H. Removing the barrier to the calculation of activation energies. J. Chem. Phys. 2016, 145, 13410 .

(16) Piskulich, Z. A.; Mesele, O. O.; Thompson, W. H. Activation energies and beyond. J. Phys. Chem. A. 2019, 123, 71857194.

(17) Piskulich, Z. A.; Thompson, W. H. Examining the role of different molecular interactions on activation energies and activation volumes in liquid water. J. Chem. Theory Comput. 2021, 17, 2659-2671.

(18) Piskulich, Z. A.; Thompson, W. H. The activation energy for water reorientation differs between IR pump-probe and NMR measurements. J. Chem. Phys. 2018, 149, 164504 .

(19) Piskulich, Z. A.; Laage, D.; Thompson, W. H. Activation energies and the extended jump model : How temperature affects reorientation and hydrogen-bond exchange dynamics in water. J. Chem. Phys. 2020, 153, 074110.

(20) Piskulich, Z. A.; Mesele, O. O.; Thompson, W. H. Removing the barrier to the calculation of activation energies: Diffusion coefficients and reorientation times in liquid water. J. Chem. Phys. 2017, 147, 134103.
(21) Piskulich, Z. A.; Thompson, W. H. The dynamics of supercooled water can be predicted from room temperature simulations The dynamics of supercooled water can be predicted from room temperature simulations. J. Chem. Phys. 2020, 152, 074505.

(22) Mendis, C.; Piskulich, Z. A.; Thompson, W. H. Tests of the Stokes-Einstein relation through the shear viscosity activation energy of water. J. Phys. Chem. B. 2019, 123, 5857-5865.

(23) Piskulich, Z. A.; Thompson, W. H. On the temperature dependence of liquid structure. J. Chem. Phys. 2020, 152, 011102.

(24) Piskulich, Z. A.; Mesele, O. O.; Thompson, W. H. Expanding the calculation of activation volumes: Self-diffusion in liquid water. J. Chem. Phys. 2018, 148, 134105.

(25) Piskulich, Z. A.; Thompson, W. H. Temperature dependence of the water infrared spectrum: Driving forces, isosbestic points, and predictions. J. Phys. Chem. Lett. 2020, 11, 7762-7768.

(26) Piskulich, Z. A.; Laage, D.; Thompson, W. H. On the role of hydrogen-bond exchanges in the spectral diffusion of water. J. Chem. Phys. 2021, 154, 064501.

(27) Borkowski, A. K.; Piskulich, Z. A.; Thompson, W. H. Examining the Hofmeister series through activation energies: Water diffusion in aqueous alkali-halide solutions. J. Phys. Chem. B 2021, 125, 350-359.

(28) Katiyar, A.; Thompson, W. H. Temperature dependence of peptide conformational equilibria from simulations at a single temperature. J. Phys. Chem. A 2021, 125, 2374-2384.

(29) Piskulich, Z. A.; Laird, B. B. Molecular simulations of phase equilibria and transport properties in a model $\mathrm{CO}_{2}$ ? expanded lithium perchlorate electrolyte. J. Phys. Chem. B 2021, 125, 9341?9349. 
(30) Berendsen, H. J.; Grigera, J. R.; Straatsma, T. P. The missing term in effective pair potentials. J. Phys. Chem 1987, 91, 6269-6271.

(31) Izadi, S.; Onufriev, A. V. Accuracy limit of rigid 3-point water models. J. Chem. Phys. 2016, 145, 074501.

(32) Abascal, J. L.; Vega, C. A general purpose model for the condensed phases of water: TIP4P/2005. J. Chem. Phys. 2005, 123, 234505.

(33) Horn, H. W.; Swope, W. C.; Pitera, J. W.; Madura, J. D.; Dick, T. J.; Hura, G. L.; Head-Gordon, T. Development of an improved four-site water model for biomolecular simulations: TIP4P-Ew. J. Chem. Phys. 2004, 120, 9665-9678.

(34) Jorgensen, W. L.; Chandrasekhar, J.; Madura, J. D.; Impey, R. W.; Klein, M. L. Comparison of simple potential functions for simulating liquid water. J. Chem. Phys. 1983, 79, 926-935.

(35) Price, D. J.; Brooks, C. L. A modified TIP3P water potential for simulation with Ewald summation. J. Chem. Phys. 2004, 121, 10096-10103.

(36) Wu, Y.; Tepper, H. L.; Voth, G. A. Flexible simple point-charge water model with improved liquid-state properties. J. Chem. Phys. 2006, 124, 024503.

(37) Tainter, C. J.; Pieniazek, P. A.; Lin, Y. S.; Skinner, J. L. Robust three-body water simulation model. J. Chem. Phys. 2011, $134,184501$.

(38) Tainter, C. J.; Shi, L.; Skinner, J. L. Reparametrized E3B (explicit three-body) water model using the TIP4P/2005 model as a reference. J. Chem. Theory Comput. 2015, 11, 2268-2277.

(39) Petersen, C.; Tielrooij, K. J.; Bakker, H. J. Strong temperature dependence of water reorientation in hydrophobic hydration shells. J. Chem. Phys. 2009, 130, 214511.
(40) Nicodemus, R. A.; Corcelli, S. A.; Skinner, J. L.; Tokmakoff, A. Collective hydrogen bond reorganization in water studied with temperature-dependent ultrafast infrared spectroscopy. J. Phys. Chem. B. 2011, 115, 5604-5616.

(41) Lang, E.; Lüdemann, H.-D. Pressure and temperature dependence of the longitudinal proton relaxation times in supercooled water to $87^{\circ} \mathrm{C}$ and 2500 bar. J. Chem. Phys. 1977, 67, 718-723.

(42) Stirnemann, G.; Laage, D. On the origin of the non-Arrhenius behavior in water reorientation dynamics. J. Chem. Phys. 2012, $13 \%, 031101$.

(43) Woolf, L. A. Tracer diffusion of tritiated water (THO) in ordinary water $\left(\mathrm{H}_{2} \mathrm{O}\right)$ under pressure. J. Chem. Soc., Faraday Trans. 1 1975, 71, 784-796.

(44) Krynicki, K.; Green, C. D.; Sawyer, D. W. Pressure and Temperature Dependence of Self-diffusion in Water. Faraday Discuss. Chem. Soc. 1978, 66, 199-208.

(45) Gillen, K. T.; Douglass, D. C.; Hoch, M. J. Self-diffusion in liquid water to $-31^{\circ} \mathrm{C}$. $J$. Chem. Phys. 1972, 57, 5117-5119.

(46) Pruppacher, H. R. Self-diffusion coefficient of supercooled water. J. Chem. Phys. 1972, 56, 101-107.

(47) Vartia, A. A.; Mitchell-Koch, K. R.; Stirnemann, G.; Laage, D.; Thompson, W. H. On the reorientation and hydrogen-bond dynamics of alcohols. $J$. Phys. Chem. B 2011, 115, 12173-12178.

(48) Kwak, K.; Rosenfeld, D. E.; Fayer, M. D. Taking apart the two-dimensional infrared vibrational echo spectra: More information and elimination of distortions. $J$. Chem. Phys. 2008, 128, 204505.

(49) Lawrence, C. P.; Skinner, J. L. Ultrafast infrared spectroscopy probes hydrogenbonding dynamics in liquid water. Chem. Phys. Lett. 2003, 369, 472-477. 
(50) Gallo, P.; Amann-Winkel, K.; Angell, C. A.; Anisimov, M. A.; Caupin, F.; Chakravarty, C.; Lascaris, E.; Loerting, T.; Panagiotopoulos, A. Z.; Russo, J. et al. Water: A tale of two liquids. Chem. Rev. 2016, 116, 7463-7500.

(51) Palmer, J. C.; Poole, P. H.; Sciortino, F.; Debenedetti, P. G. Advances in computational studies of the liquid-liquid transition in water and water-like models. Chem. Rev. 2018, 118, 9129-9151.

(52) Hestand, N. J.; Skinner, J. L. Perspective: Crossing the Widom line in no man's land: Experiments, simulations, and the location of the liquid-liquid critical point in supercooled water. J. Chem. Phys. 2018, 149, 140901.

(53) Ni, Y.; Hestand, N. J.; Skinner, J. L. Communication: Diffusion constant in supercooled water as the Widom line is crossed in no man's land. J. Chem. Phys. 2018, $148,191102$.

(54) Xu, Y.; Petrik, N. G.; Smith, R. S.; Kay, B. D.; Kimmel, G. A. Growth rate of crystalline ice and the diffusivity of supercooled water from 126 to 262 K. Proc. Natl. Acad. Sci. U.S.A. 2016, 113, 1492114925.

(55) Evans, M. G.; Polanyi, M. Some applications of the transition state method to the calculation of reaction velocities, especially in solution. Trans. Faraday Soc. 1935, 31, 875-894.

(56) Ladanyi, B. M.; Hynes, J. T. Transitionstate solvent effects on atom transfer rates in solution. J. Am. Chem. Soc 1986, 108, 585-593.

(57) Reichardt, C. Solvents and Solvent Effects in Organic Chemistry; VCH: New York, 1990.

(58) Harris, K. R.; Woolf, L. A. Pressure and temperature dependence of the self diffusion coefficient of water and oxygen-18 water. J. Chem. Soc., Faraday Trans. 1 1980, 76, 377-385.

(59) Harris, K. R.; Newitt, P. J. Self-diffusion of water at low temperatures and high pressure. J. Chem. Eng. Data 1997, 42, 346-348.

(60) Ewell, R. H.; Eyring, H. Theory of the viscosity of liquids as a function of temperature and pressure. J. Chem. Phys. 1937, 5, 736-744.

\section{Biographies}

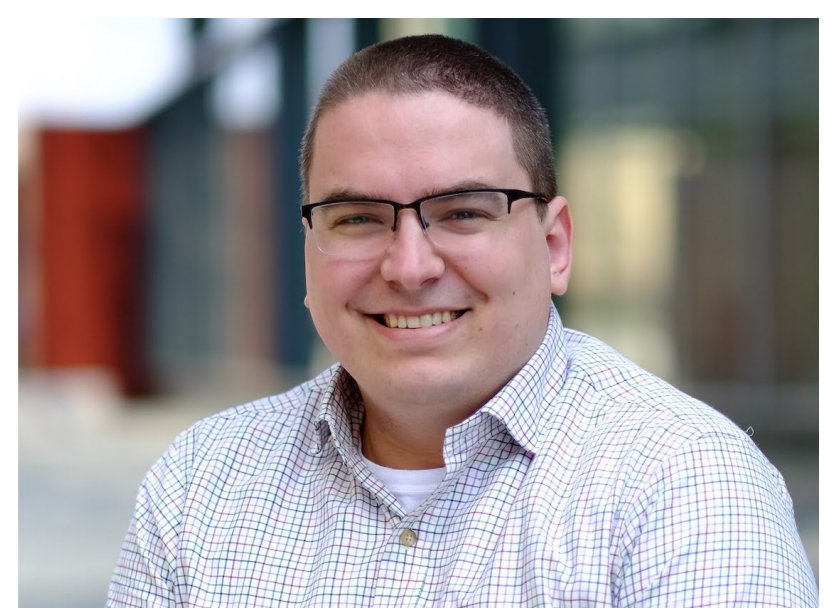

Zeke A. Piskulich is a postdoctoral research associate in the group of Prof. Qiang Cui in the Boston University Department of Chemistry. His research currently focuses on the interaction between nanoparticles and lipid membranes. He received his B.S. degree in Physics from the University of Missouri, Columbia, in 2017. Zeke received his Ph.D. in Chemistry from the University of Kansas in 2021 where he worked with Profs. Ward H. Thompson and Brian B. Laird developing a fluctuation theory approach for calculating activation energies. During his Ph.D. Zeke was both an NSF graduate research fellow and a Lindau young scientist. 


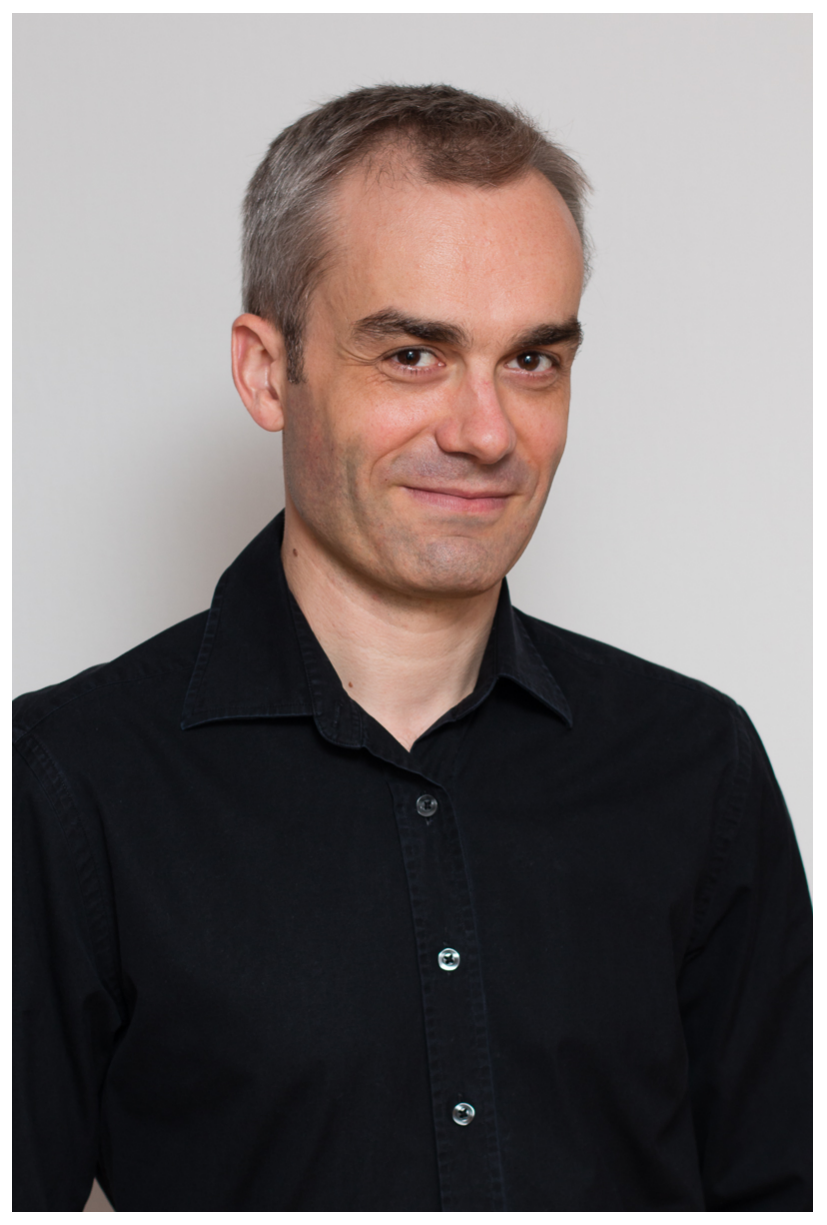

Damien Laage is a CNRS Directeur de Recherche and a Professeur attaché at Ecole Normale Supérieure (ENS) in Paris. His research interests focus on chemical reactivity in solutions, at interfaces and in biochemical environments. He received his Ph.D. degree from ENS and Sorbonne Univ. in 2001 and he was a postdoctoral fellow at ETH Zrich in 2002, before joining ENS.

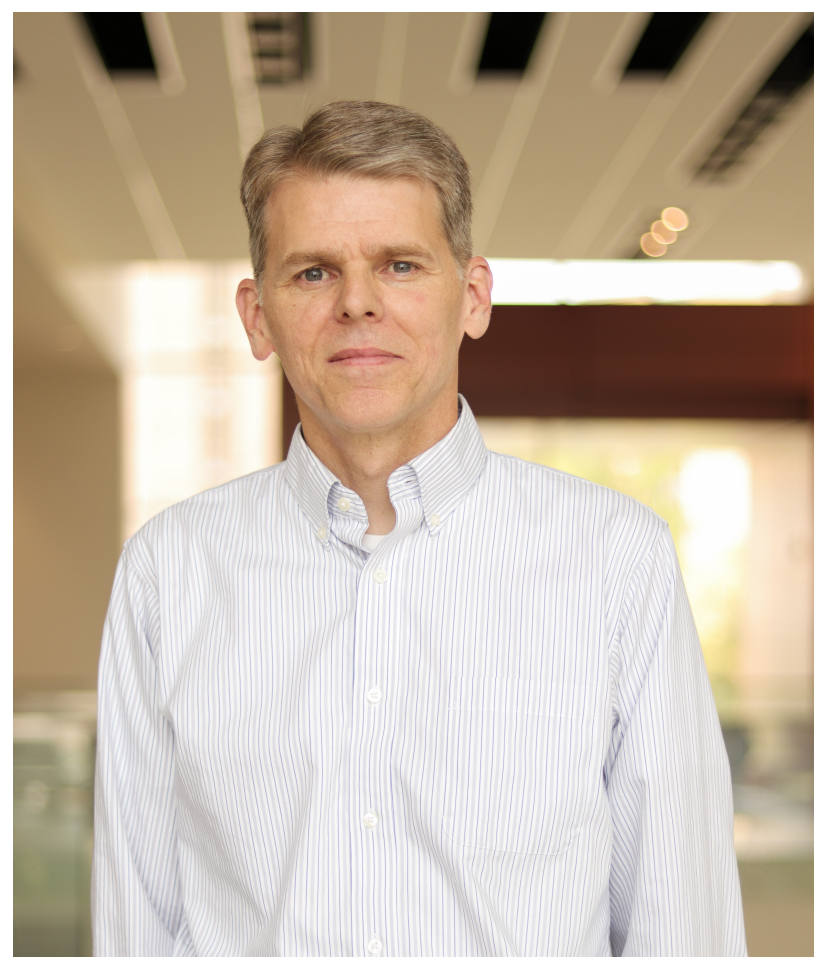

Ward H. Thompson is Professor of Chemistry at the University of Kansas. His research focuses on understanding chemical dynamics in liquids, with particular interest in porous materials, hydrogen-bonding, and vibrational spectroscopy. He received his B.S. degree in Physics from Oklahoma State University in 1991 and his Ph.D. in Chemistry from the University of California, Berkeley in 1996. He worked as a postdoctoral associate at the University of Colorado, Boulder before joining the University of Kansas as a faculty member in 2001. 
Graphical TOC Entry

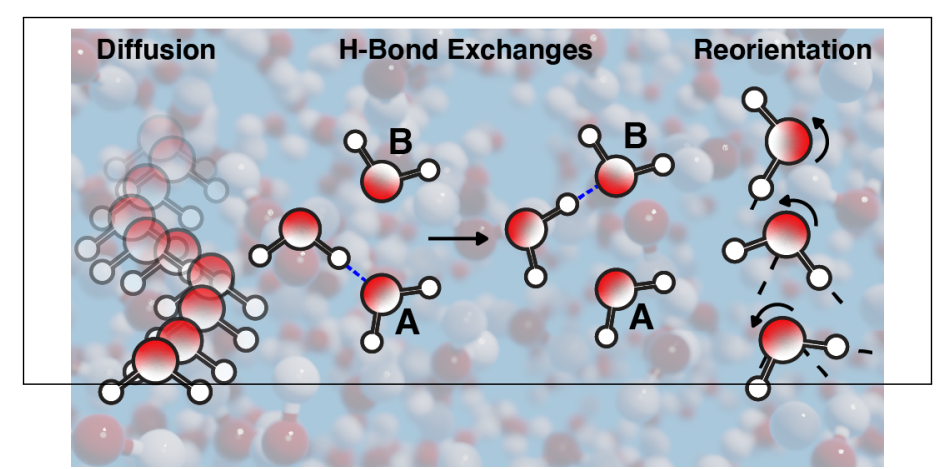

